Volume 12, No. 2, March-April 2021

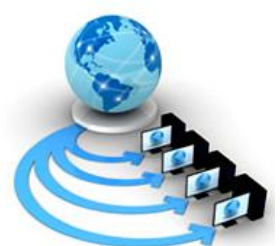

International Journal of Advanced Research in Computer Science

RESEARCH PAPER

Available Online at www.ijarcs.info

\title{
LEHER - LOW-COST ENHANCED HYDRO-NAVIGATION SYSTEM ENSURING REAL-TIME MONITORING OF SHIPS WITH THE HELP OF RASPBERRY PI, ROCKBLOCK 9603 SATELLITE COMMUNICATION MODULE AND A COUPLE OF OTHER SENSORS
}

\author{
Prithwijit Das \\ Department of Computer Science \& Engineering \\ JIS College of Engineering \\ Kalyani, Nadia, W.B., India \\ Siddhanta Dutta \\ Department of Computer Science \& Engineering \\ JIS College of Engineering \\ Kalyani, Nadia, W.B., India
}

\author{
Deepankar Kumar \\ Department of Computer Science \& Engineering \\ JIS College of Engineering \\ Kalyani, Nadia, W.B., India \\ Shuva Mandal \\ Department of Computer Science \& Engineering \\ JIS College of Engineering \\ Kalyani, Nadia, W.B., India
}

\author{
Sumanta Chatterjee \\ Department of Computer Science \& Engineering \\ JIS College of Engineering \\ Kalyani, Nadia, W.B., India
}

\begin{abstract}
Wrecks are by and large caused because of carelessness in route. As we probably are aware route includes human move in this way mishappen is consistently an opportunity. To decrease this, it is our answer to make a self-governing route-able boat utilizing 'Global Positioning System' support. Latitude and longitude coordinates will allow the boat to decide its precise area on the water body. The coordinates of the destination will be pre-loaded before the flight of the boat. A fanciful way will be resolved between the boat's area and the last area. A magnetometer sensor will be utilized to clarify the geological area of the boat itself to guide the navigation. If there should arise an occurrence of any capricious snag shows up on the radar the boat will be prepared to make an impermanent way to stay away from the deterrent. In the wake of passing the impediment, it will keep on after the recently decided way by following back to the directions that were fixed during setting the last objective directions. The Rockblock 9603 Satellite Communication Module is a special GPS module that can be utilized to send short burst information to the Iridium satellite and back to earth. We will utilize this module to set up a solid association of the boat to the control room. Besides, this module will help us cause the boat to explore independently, with no human intercession.
\end{abstract}

Keywords: Raspberry Pi, Magnetometer, Global Positioning System, Satellite Communication, Autonomous Navigation

\section{INTRODUCTION}

The early Pacific Polynesians were quick to travel significant distances on the ocean utilizing the movement of the stars or the situation of certain untamed life species, the size of the waves was utilized to discover the way starting with one island then onto the next. Utilizing this technique was very little advantageous as it was not steady for the turn of Earth. As an answer, it was found that the Polaris in the North sky doesn't appear to move. The North Star is the most straightforward technique for discovering the course at that point. Today, all huge boats depend on the Global Positioning System. Marine GPS collectors don't show roads they give longitude and scope and commonly show Maps of any close by coastlines, harbors, beacons, and so on Numerous likewise show the estimated profundity of the water too. Numerous marine GPS units currently have a man over the edge button. On the off chance that any individual falls over the edge, anybody among the group individuals or the chief presses that button. The GPS unit at that point denotes that spot in the sea and regularly shows it as a directional bolt highlighting it alongside the distance perusing to that detect that allows the skipper to turn the boat around and get back to where the individual fell into the water. Notwithstanding, numerous boats even now convey a handheld instrument used to gauge points between the sun and moon a skyline as a back up to their GPS alongside an off base wristwatch and route tables were capable of guides. Utilizing GPS information, the moving of the boat is made selfsufficient.

\section{RELATED WORKS}

In [1] the creators intend to follow the vehicle's area for different security reasons utilizing Rasberry pi as the CPU. This task center around keeping the vehicle on the correct course on its excursion starting with one point then onto the next. As this gadget has admittance to the area of the vehicle, designers additionally permit the clients to follow their vehicle if it's absent. If there should be an occurrence of the missing proprietor has choices like utilizing a secret code/key to on or off the motor controlling the fuel infusion utilizing an electronic solenoid valve. This gadget is additionally fit for following bicycles and turning the start off if there should be an 
occurrence of missing the guidance of the bicycle proprietor and giving bicycle area arranges. The correspondence between the vehicle and the proprietor is conceivable on account of the GSM module utilized in the circuit.

In [5] the writers expound on GPS in a type of route and GPS beacon. For route, this undertaking utilizes pc to get to the situation of a client and it interfaces utilizing google maps. The GPS beacon noticed the topographical organizes and advances them to the client through SMS and email.

In [8], authors expect to find vehicles under basic conditions, for example, mishaps. It utilizes a GPS module to take note of the directions and save in AT89s52 microcontroller alongside different sensors like fire discovery for blast and an infrared sensor for movement location. They expect to oversee traffic later on with the GPS information, after accepting it from the vehicle. This venture likewise expects to zero in on decreasing the danger of utilizing vehicles for bomb blasts by presenting a bomb finder in the circuit. The executive framework is likewise a point of convergence of this undertaking where it intends to interface every one of the vehicles and look after them.

In [10], a submerged vehicle is included that can do different exercises in the re-position of people working distantly to use in high strain circumstances where human existence can be in danger. This can be utilized to keep up the biological system by social affair different data like temperature and pressing factor at one point in the water. This undertaking is likewise in the uncertainty of legitimate remote correspondence because of the presence of water medium so as an answer they keep it wired.

\section{METHODOLOGY}

Here, we have proposed a methodology towards making the route of boats computerized, with no human mediation. Through this technique, we have attempted to incorporate the control unit of the boat. The dynamic capacity of the independent route framework is upgraded with the information got from various kinds sensors utilized for devoted purposes, as follows:

\section{A. Direction Analysis}

To begin the crossing, the boat needs to decide the heading it is confronting. The magnetometer sensor will offer the simple benefit to the Raspberry pi, where the current heading is found through the introduced program. If it's confronting a misguided course, the propellor and the rudder are impelled to carry it to the necessary heading. Except if it arrives at the proper bearing, the framework would not allow the boat to push ahead. Then again, if turning is absurd, i.e., the information from ultrasonic sensors isn't adequately reasonable to permit turning, the framework upholds the boat to move the converse way. At the point when it is agreeable enough for the boat to turn, the framework instates the standard crossing.

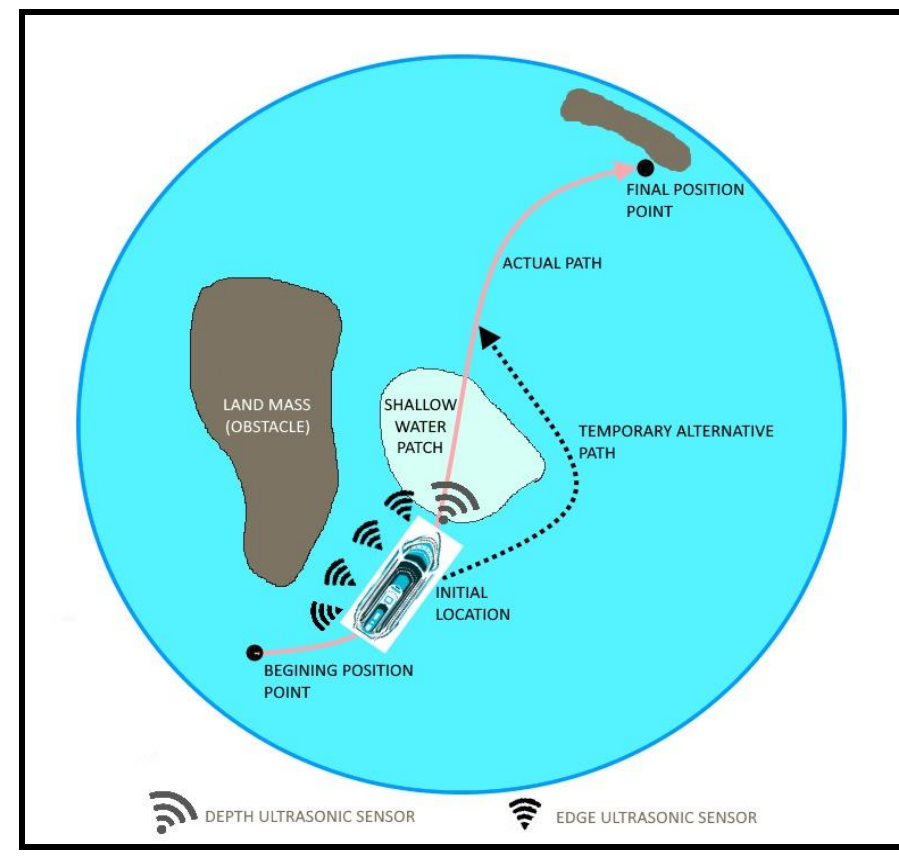

Figure 1. Anti-Collision Traversal

\section{B. Avoiding Obstacles}

One of the significant dangers to ships, cruising in the sea, is the uncommon deterrents. These should be kept away from to protect the boat just as the travelers or products inside it. Here, obstructions might be arranged into two sorts: a) Static These are the shallow patches of land, coasting ice sheets, or a float of extensive size; b) Dynamic - These can go from little boats to enormous boats. On the off chance that any such circumstance is experienced, the information ceaselessly being brought from the ultrasonic sensors will make the framework mindful to execute a crisis hostile to impact crossing. The standard crossing will be halted except if the hindrance is overwhelmed totally. Besides, the framework will make the boat sidestep the deterrent where the development will be altogether guided by the information got from ultrasonic sensors.

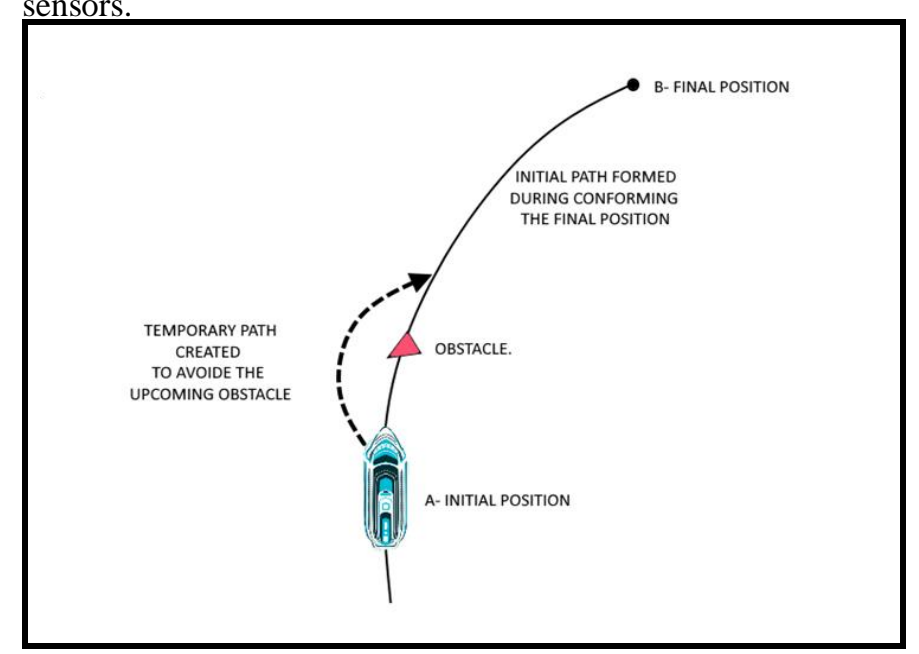

Figure 2. Obstacle Avoidance

\section{Real-time Monitoring}

While cruising on profound seas, boats may deal with various issues, for example, - discharged gas tank, unexpected separate, seize, and so forth. Such issues should be accounted for in the control space for sure-fire reinforcement and salvage. Presenting a dependable two-route correspondence between the 
boat and the control room is the most ideal approach to partner such a circumstance. The Rockblock 9603 Satellite Communication Module satisfies the requirement for satellite correspondence. It is a handset that can be utilized to send short burst information to the Iridium satellite group of stars and back to earth. It has three particular highlights - a) It can get the current GPS area from satellite, b) Send information to the satellite which can be additionally transferred to the cloud, c) It can be utilized to speak with different boats utilizing a similar correspondence module. At the point when the framework sends the current area of the boat to the satellite at standard stretches lastly unloaded into a cloud worker, the live area of the boat can be followed from the control room. Through this framework, continuous checking of a boat conveying travelers or products can be accomplished. A disparity while observing will create a crisis alert in the control room and consequently, decline the postponement in sending reinforcement.

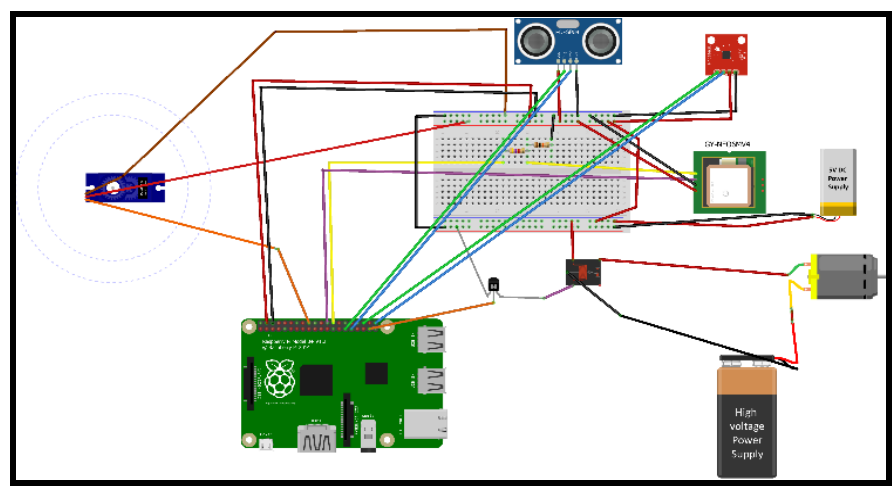

Figure 3. Circuit Diagram of the Proposed Technology

\section{Autonomous Traversal Process}

A self-sufficient framework for crossing alludes to a driverless route. For a boat, to arrive at its objective, the Captain observes a standard course map comprising of a few designated spots. In this robotized route framework, a CSV document is transferred to the Raspberry $\mathrm{Pi}$. The document contains the latitudinal and longitudinal directions of the designated spots, in an even configuration, that should be navigated to arrive at the objective. These designated spots are pre-characterized to guarantee that a self-governing boat takes the most limited possible way. These designated spots will be around $2-3 \mathrm{Km}$ from one another. The control unit utilizes the Rockblock 9603 Satellite Communication Module to recover the current latitudinal and longitudinal directions. It contrasts the information and the gave CSV record and decides the heading towards which the boat should confront.

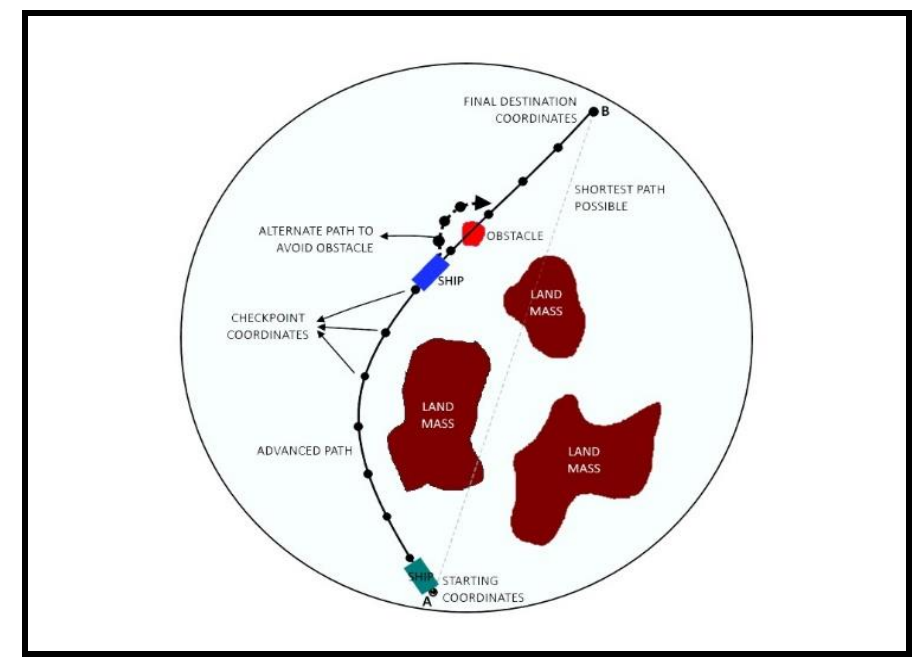

Figure 4. Automated Navigation

After bearing examination by the magnetometer, the boat is good to go to begin its excursion. At that point, a persistent cycle of getting the current area and incitation of the propellor is finished. Not long after arriving at the following designated spot, the interaction of bearing investigation and crossing is rehashed until the last designated spot, i.e., the objective is reached. Then again, the deterrent recognition and aversion program will run close by to keep away from any mishap or crash. The blend of these two projects will make the excursion more secure and more solid.

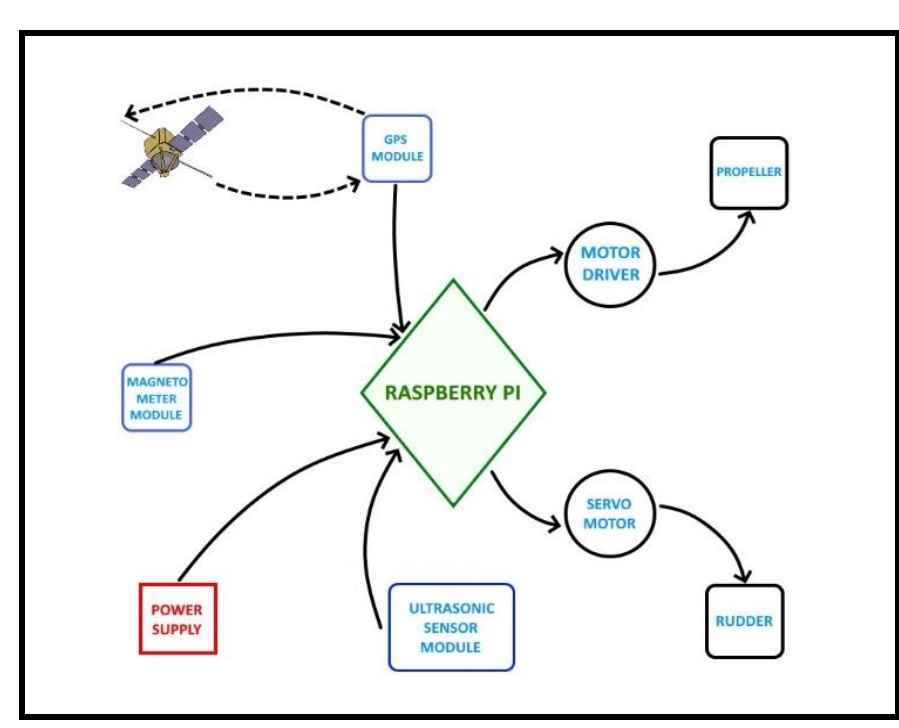

Figure 5. Block Diagram of the Entire System

As clarified in fig.5, the Raspberry Pi board is the focal control unit which is having admittance to the next sensor hubs. It requires a persistent $5 \mathrm{~V}$ DC power supply. The magnetometer gives the specific course of the boat, while the SONAR helps in distinguishing diverse static and non-static impediments. The GPS module is utilized to get the latitudinal and longitudinal directions of the boat. This module is additionally answerable for building up a two-way satellite correspondence, prompting continuous checking of that transport. Consequently, comparing to a specific arrangement of information, the Raspberry Pi conveys a message to the engine driver and carries out a speed control to the propeller. Then again, it can likewise impel the rudder with a servo engine. 


\section{RESUlts AND Discussions}

The previously mentioned innovation will give a continuous observing office of boats cruising in profound seas to the control room. The boats will cruise their allocated objective with an independent route module. During this crossing, a few obstacles can be experienced, similar to a) Determining its heading, b) To which bearing it needs to cruise, c) When to go ahead, d) To what distance should it sail a specific way, e) How to handle any deterrent going ahead of its way, be it a shallow pitch of land or another boat, f) Sending its live area to control room. The proposed self-sufficient route module is fit for tackling the previously mentioned issues in an interesting and minimal technique. It utilizes a few sensors and satellite correspondence to satisfy the destinations.

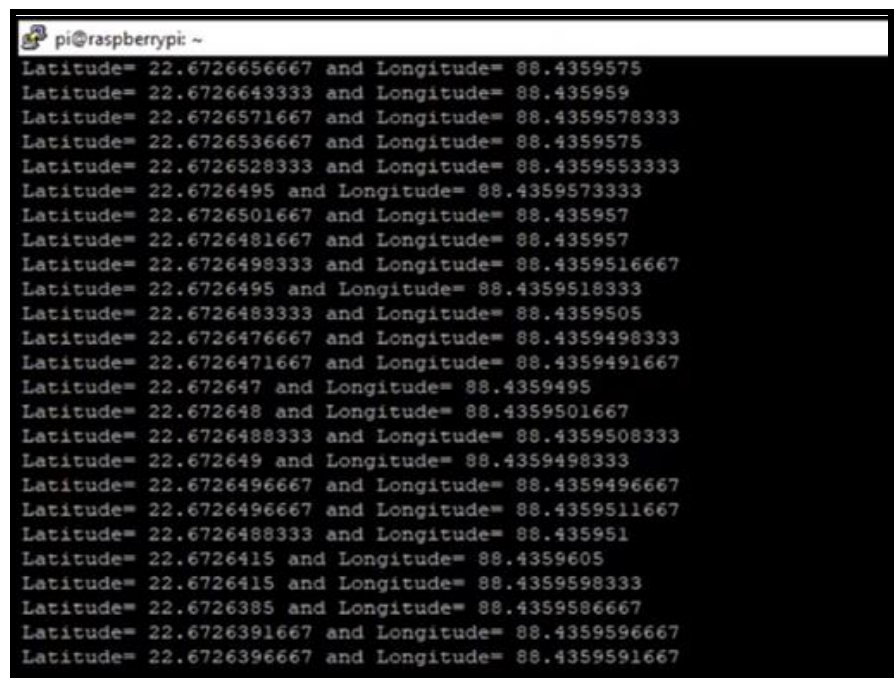

Figure 6. Live feed of Current Location fetched from GPS Module

The solitary delay that happens in this framework, is simply the correspondence method. Since there could be no different alternatives, we needed to acknowledge the deferral brought about by satellite correspondence. The Raspberry $\mathrm{Pi}$ is exceptionally proficient in performing such complex tasks parallelly.

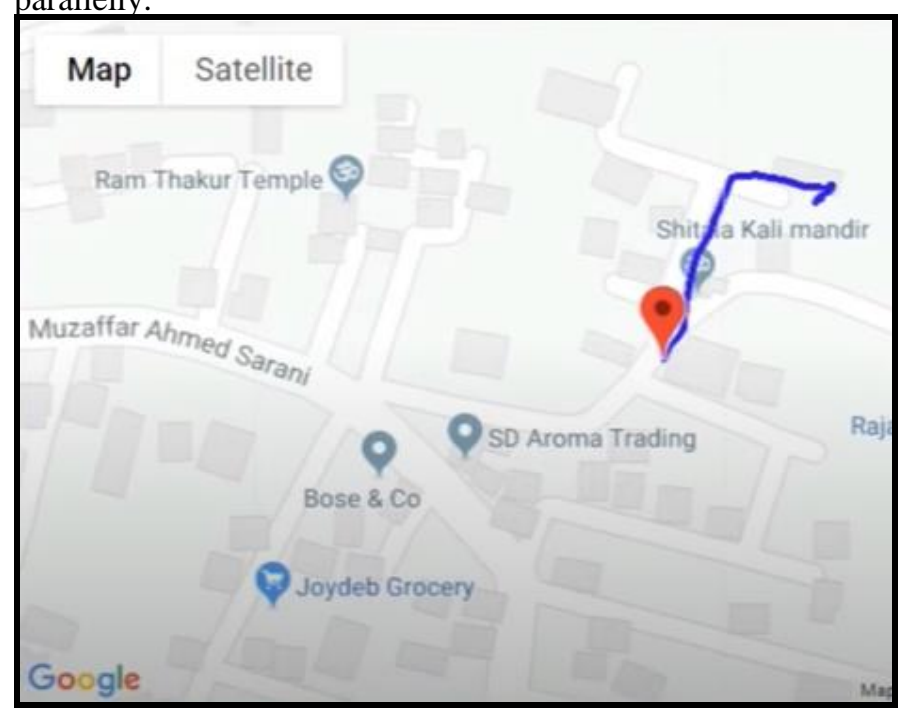

Figure 7. Test results of Real-Time Monitoring through Google Map API

Here, the information got from the sensors, all in all, assume a significant part in the dynamic, for the following move. The deduction drawn from this information is utilized to incite the rudder and the propeller/s. In this way, shaping a total sensor organization and Raspberry pi being treated as the 'heart' of the whole framework.

\section{Conclusions}

A GPS route framework consistently assists with bettering course the board framework. It will decide the way that outdated every one of the impediments to arrive at the objective. This framework will make the boat navigate the briefest way from source to destination. So the fuel utilization for the boats can be controlled. Utilizing GPS we generally get an ongoing area of these boats including the scopes and longitudes. In this way, if any boat separates halfway, we can undoubtedly find it. A firefighting ship with a GPS route framework can be controlled distantly from a space a long way from the fundamental fire point. So this will diminish the no. of losses brought about by manual obliviousness of fundamental realities. What's more, at last with computerization through python programming, these boats can be allocated with objective directions to explore self-sufficiently, with no human mediation. A GPS-empowered correspondence framework can likewise give a quicker SOS sign to the control room, on the off chance that any reinforcement is required.

\section{REFERENCES}

[1] Ds. Rani and K. Radhika Reddy Associate, "International Journal of Computer Science and Mobile Computing RASPBERRY PI BASED VEHICLE TRACKING AND SECURITY SYSTEM FOR REAL-TIME APPLICATIONS," Int. J. Comput. Sci. Mob. Comput., vol. 5, no. 7, pp. 387-393, 2016, [Online]. Available: www.ijcsmc.com.

[2] P. Pendam, J. Khan, R. Solanki, and R. Kaur Kahlon, Prof, "Location Monitoring System using Raspberry PI," vol. 2, no. 08, pp. 357-360, 2016.

[3] H. Krishna, M. Narayanan, and F. Barucha, "Smart and Autonomous Cleaning Robot," vol. 6, no. 4, pp. 452-458, 2018.

[4] S. Dana, "GPS Tracking System Architecture," p. 1213337, 2015, [Online]. Available: http://www.roadmatics.com/index.php/blog/37-gpstracking-system-architecture.

[5] Y. Khraisat, M. Al-Khateeb, Y. Abu-Alreesh, A. Ayyash, and O. Lahlouh, "GPS Navigation and Tracking Device," iJIM, vol. 5, pp. 39-41, 2011, DOI: 10.3991/ijim.v5i4.1781.

[6] X. Zhu, S. Chen, and T. Li, "Research on Magnetic localization method of underwater Magnetometer in Single component," IOP Conf. Ser. Earth Environ. Sci., vol. 237, p. 32034, 2019, DOI: 10.1088/17551315/237/3/032034.

[7] M. Naveenkrishna and S. Jayanthy, "Real-time vehicle tracking and monitoring using raspberry pi," vol. 10, pp. 15259-15263, 2015.

[8] S. Verma, A. S. Jamwal, S. Chauhan, and S. Mohanty, "Vehicle Tracking System Using GPS and GSM," Lect. Notes Electr. Eng., vol. 668, pp. 779-786, 2021, DOI: 10.1007/978-981-15-5341-7_59.

[9] J. Borenstein and Y. Koren, "Obstacle avoidance with ultrasonic sensors," Robot. Autom. IEEE J., vol. 4, pp. 213-218, 1988, DOI: 10.1109/56.2085.

[10] G. D. Priya and I. Harish, "Raspberry PI Based Underwater Vehicle for Monitoring Aquatic Ecosystem," Int. J. Eng. Trends Appl., vol. 2, no. 2, pp. 65-71, 2015, [Online]. Available: www.ijetajournal.org. 
Prithwijit Das et al, International Journal of Advanced Research in Computer Science, 12 (2), March-April 2021,45-49

[11] A. Schubert, M. Kurowski, M. Gluch, O. Simanski, and T. Jeinsch, "Manoeuvring Automation towards Autonomous Shipping," 2018, DOI: 10.24868/ISSN.26318741.2018.020.
[12] A. Barnagarwala and A. Buriwala, "Vehicle Tracking and Monitoring System Using Gps and Gsm/Gprs," Int. J. Eng. Sci. Res. Technol., vol. 6, no. 12, pp. 17-21, 2017. 